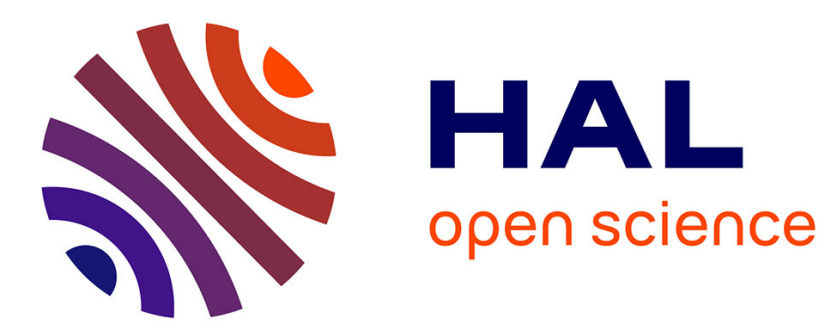

\title{
Flow Enhancement due to Elastic Turbulence in Channel Flows of Shear Thinning Fluids
}

Hugues Bodiguel, Julien Beaumont, Anaïs Machado, Laetitia Martinie, Hamid Kellay, Annie Colin

\section{- To cite this version:}

Hugues Bodiguel, Julien Beaumont, Anaïs Machado, Laetitia Martinie, Hamid Kellay, et al.. Flow Enhancement due to Elastic Turbulence in Channel Flows of Shear Thinning Fluids. Physical Review Letters, 2015, 114 (2), pp.028302 (1-5). 10.1103/PhysRevLett.114.028302 . hal-01105020

\author{
HAL Id: hal-01105020 \\ https://hal.science/hal-01105020
}

Submitted on 19 Jan 2015

HAL is a multi-disciplinary open access archive for the deposit and dissemination of scientific research documents, whether they are published or not. The documents may come from teaching and research institutions in France or abroad, or from public or private research centers.
L'archive ouverte pluridisciplinaire HAL, est destinée au dépôt et à la diffusion de documents scientifiques de niveau recherche, publiés ou non, émanant des établissements d'enseignement et de recherche français ou étrangers, des laboratoires publics ou privés. 


\title{
Flow Enhancement due to Elastic Turbulence in Channel Flows of Shear Thinning Fluids
}

\author{
Hugues Bodiguel, ${ }^{1}$ Julien Beaumont, ${ }^{1}$ Anaïs Machado, ${ }^{1}$ Laetitia Martinie, ${ }^{1}$ Hamid Kellay, ${ }^{2}$ and Annie Colin ${ }^{1,3}$ \\ ${ }^{1}$ Université de Bordeaux, CNRS, Solvay, LOF UMR 5258, Pessac 33600, France \\ ${ }^{2}$ Université de Bordeaux, CNRS, LOMA UMR 5586, Talence 33501, France \\ ${ }^{3}$ Université de Bordeaux, CRPP, UPR 8641, Pessac 33600, France
}

(Received 12 February 2014; revised manuscript received 22 September 2014; published 15 January 2015)

\begin{abstract}
We explore the flow of highly shear thinning polymer solutions in straight geometry. The strong variations of the normal forces close to the wall give rise to an elastic instability. We evidence a periodic motion close the onset of the instability, which then evolves towards a turbulentlike flow at higher flow rates. Strikingly, we point out that this instability induces genuine drag reduction due to the homogenization of the viscosity profile by the turbulent flow.
\end{abstract}

DOI: 10.1103/PhysRevLett.114.028302

PACS numbers: $83.80 . \mathrm{Rs}, 47.50 . \mathrm{Gj}, 83.50 . \mathrm{Ha}$

Viscoelastic polymer solutions are characterized by a relaxation time $\lambda$ required by the polymer molecules to adjust to changes in the flow conditions. This time is at the origin of purely elastic instabilities observed at very low Reynolds numbers with no counterpart in pure Newtonian fluids. In shear flows, purely elastic instabilities arise due to stress tensor anisotropy inducing a destabilizing net force perpendicularly to curved streamlines [1-3]. These instabilities can lead to purely elastic turbulence [4-6].

In straight channels, theoretical studies demonstrate that the viscosimetric properties of the fluid play a major role in the stability of the flow. Plane Couette flows [7] and Poiseuille flows [8] of Oldroyd-B fluids exhibit a nonlinear subcritical elastic instability although the base flow is linearly stable [9]. Recent experiments [10,11] have shown that finite amplitude perturbation creates curved streamlines that drive the instability. In contrast, channel flows of highly shear thinning fluids [12-14] are theoretically linearly unstable. The instability is driven in this case by the strong variations of both normal stress and viscous dissipation in the shear direction. An extreme situation is obtained with shear banding fluids, where the interface bears an unbalanced normal stress [14]. This leads to an interfacial instability which has been observed experimentally [15]. A similar mechanism is theoretically expected without shear banding but requires a strong gradient of shear rate [13]. At this stage, channel flow stability of such liquids has not been studied experimentally, although shear thinning is a very common feature of elastic fluids.

In this Letter, we focus on highly shear thinning elastic polymer solutions with no shear banding flowing in straight channels. At low flow rates, no velocity fluctuations are observed. At higher flow rates, the power spectrum density of the velocity fluctuations displays a distinct peak indicating the onset of instability. The position of the peak is in agreement with theoretical predictions for highly shear thinning fluids [13]. At even higher flow rates, the fluctuations occur at all scales and the spectrum becomes broadband with a power law decay. This instability induces genuine drag reduction: viscous losses are smaller than expected from the fluid rheology. This is a remarkable result since one expects additional energy losses due to the enhancement of velocity fluctuations.

We study the flow of high molecular weight polymer solutions $\left(18 \times 10^{6} \mathrm{~g} / \mathrm{mol}\right.$ partially hydrolyzed polyacrylamide) in water at different concentrations in the semidilute regime. The global flow curves of these solutions are determined using the shear-rate-imposed mode of a rheometer (TA Instruments ARG2) in a sanded cone-andplate geometry of angle $\theta=2^{\circ}$. Figure 1 reports both the shear stress $\sigma$ and the Weissenberg number Wi $=N_{1} / 2 \sigma$ as

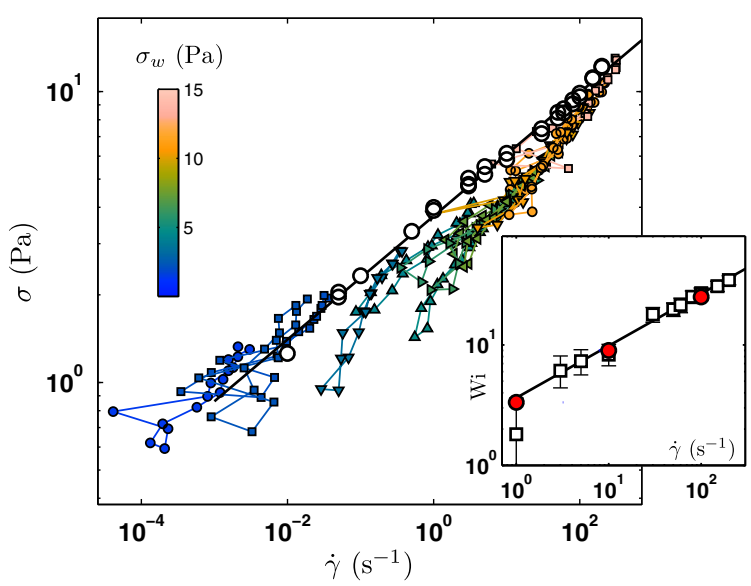

FIG. 1 (color online). Flow curve obtained by standard bulk rheometry (large open circles), and best power-low fit (straight solid line) $\sigma=3.73 \dot{\gamma}^{0.21}$, for $\dot{\gamma}<50 \mathrm{~s}^{-1}$. The small colored symbols are obtained by differentiating the velocity profiles in both 152 and $170 \mu \mathrm{m}$ width channels. The wall shear stress $\sigma_{w}$ of each experiment is indicated in the color bar. In the right axes is plotted the Weissenberg number, either from direct normal force measurement, $\mathrm{Wi}=N_{1} / 2 \sigma$ (open squares), or from characteristic time estimations $\mathrm{Wi}=\tau \dot{\gamma}$ (colored circles) using step flow experiments. The solid line stands for $\mathrm{Wi}=3.63 \dot{\gamma}^{0.43}$. 
a function of the shear rate $\dot{\gamma}$ (see Supplemental Material [16] for additional details) of the most concentrated solution $(0.4 \%) . N_{1}$ is the first normal stress difference. The fluid is highly shear thinning: $\sigma=3.73 \dot{\gamma}^{0.21}$ and $\mathrm{Wi}=3.63 \dot{\gamma}^{0.43}$. This behavior corresponds to a WhiteMetzner fluid, for which $\sigma=G \tau \dot{\gamma}$ and $N_{1}=2 G \dot{\gamma}^{2} \tau^{2}$; the relaxation time $\tau$ and the elastic modulus $G$ are power-law functions of $\dot{\gamma}$, respectively given by $\tau=3.63 \gamma^{-0.57}$ and $G=1.02 \gamma^{-0.22}$. This description is validated by independent measurements of $\tau$, assumed to be the characteristic time of the shear stress relaxation after a shear rate peak hold (see Fig. 1). The stability of channel flows of this type of fluid has been investigated theoretically [13] and found linearly unstable when the shear-thinning exponent is very low $(<0.2)$. More dilute solutions also exhibit an important shear-thinning behavior, but cannot be described using simple power law functions (see Supplemental Material [16]).

We study the flow of these solutions in homemade straight channels (length $L=5.5 \mathrm{~cm}$, height $h=1 \mathrm{~mm}$, widths $w=152$ and $170 \mu \mathrm{m}$ ) using particle image velocimetry (see Supplemental Material [16] and Ref. [18]). The canyon geometry used here is rather well approximated by two infinite parallel planes (at least close to the midplane). The main velocity component is along the channel length ( $x$ direction), and the velocity gradient is along the channel width ( $y$ direction). The typical error on the velocity for a single correlation is about $10 \%$, independently of the velocity value, since the time step is adjusted for each experiment. The mean velocity is estimated using about 1000 images; the precision is below 1\%. A differential pressure-control regulator (MFCS $4 \mathrm{C}$, Fluigent), with a range of pressure drops $\Delta P$ between 5 and 125 mbar is used. Even though the experiments are performed in rectangular channels, no vortices along the channel have been observed [19] within our experimental precision. For all the experiments reported here, the transverse velocity components $v_{y}, v_{z}$ are negligible compared to that in the flow direction $v_{x}$. Lubrication approximation is thus valid and the time-averaged shear stress is given by $\sigma_{x y}=y \partial_{x} p$, where $p$ is the pressure. Velocity profiles measured at different locations along the channel length superimposed perfectly, indicating a constant pressure gradient $\Delta P_{c} / L$. Using a Bagley correction [20], we found $\Delta P_{c}$ between 0.8 and 1 times $\Delta P$ [16]. Here, we use the wall shear stress $\sigma_{w}=\Delta P_{c} w / 2 L$ as the control parameter.

Figure 2 reports the instantaneous velocities and the time-averaged velocity profiles for the $0.4 \%$ solution at different $\sigma_{w}$. The latter are pluglike as expected for shear thinning fluids. The experiments could be separated into two regimes: for $\sigma_{w} \geq 4.86 \mathrm{~Pa}$, the velocity exhibits large fluctuations and apparent slippage at the wall which is typically $30 \%$ of the maximal velocity. For lower values of $\sigma_{w}$, the velocity fluctuations are small and the apparent slip velocity contribution to the flow is much higher (greater

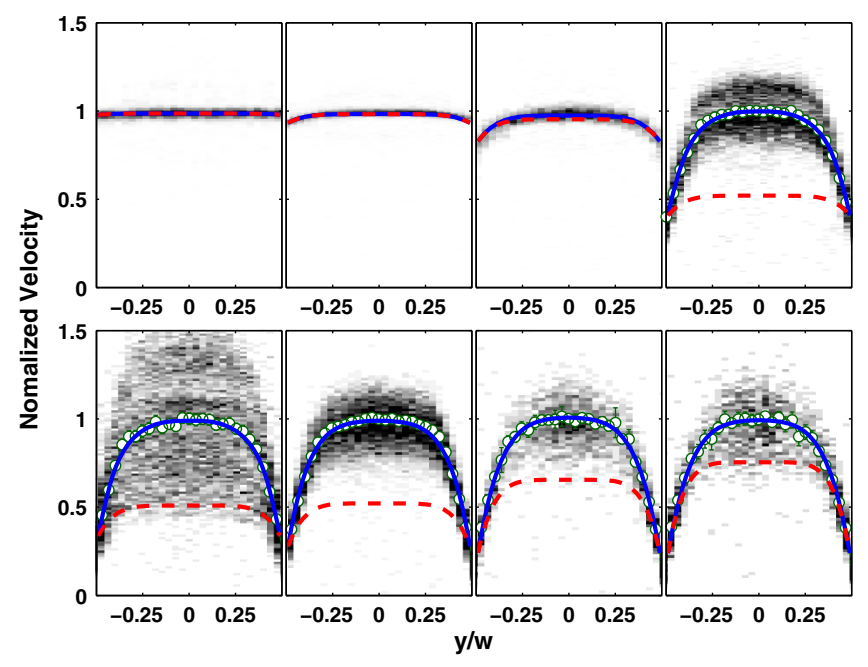

FIG. 2 (color online). Velocity profiles obtained in a channel of width $w=152 \mu \mathrm{m}$. From top left to bottom right, the values of the applied pressure drop are 10, 17.8, 26.7, 36.7, 47.5, 57.7, 80, and 90 mbar, which correspond after end-effect correction to wall shear stress $\sigma_{w}$ of $1.33,2.36,3.54,4.86,6.28,7.60,10.4$, and $11.5 \mathrm{~Pa}$. The velocity is normalized by the average maximum velocity measured in the channel center, i.e., $5.57 \times 10^{-6}$, $1.14 \times 10^{-5}, 8.22 \times 10^{-5}, 4.17 \times 10^{-4}, 9.19 \times 10^{-4}, 1.62 \times 10^{-3}$, $4.41 \times 10^{-3}, 5.43 \times 10^{-3} \mathrm{~m} / \mathrm{s}$. The mean velocity profile is displayed as circles and is superimposed on a gray-scale image which represents the velocity density probability estimated from correlation counts in a correlation box of width $5.6 \mu \mathrm{m}$. The dashed line is the velocity prediction from the liquid bulk rheological power law (see text) where the slip velocity is the single fitting parameter. The solid line represents the best fit to the data using the nonlocal fluidity model (see text).

than $80 \%$ ). The apparent slip in these velocity profiles corresponds to the presence of a very small depletion zone devoid of polymers [21]. For low $\sigma_{w}$ no velocity fluctuations are observed. The standard deviation (std) of the longitudinal velocity (Fig. 3) then increases from nearly zero to $50 \%$ of the mean velocity at higher values of $\sigma_{w}$. The variation of the std versus $\sigma_{w}$ clearly indicates the onset of an instability. The onset wall shear stress comprises between 3.5 and $4.7 \mathrm{~Pa}$ (corresponding to $3.65<\mathrm{Wi}<$ 5.8). The std goes through a maximum at $\sigma_{w}=6 \mathrm{~Pa}$ and reaches a plateau value of $30 \%$ of the mean velocity for $\sigma_{w}>7 \mathrm{~Pa}$.

We then measure the fluctuations along the $y$ direction. This is a difficult task since $v_{y}$ is very small compared to $v_{x}$. The channel is placed on a translation stage, moving in the opposite direction to the flow, with a velocity tuned manually so that the observation frame almost coincides with that of the maximum velocity. The same particles thus remain in the field of observation. Rather than image pairs, we acquire standard image sequences at $10 \mathrm{fps}$ and reconstruct the trajectories of the individual tracers using a standard particle tracking algorithm [26]. For $\sigma_{w}<4.7 \mathrm{~Pa}$, the particle trajectories are well aligned in the $x$ direction 

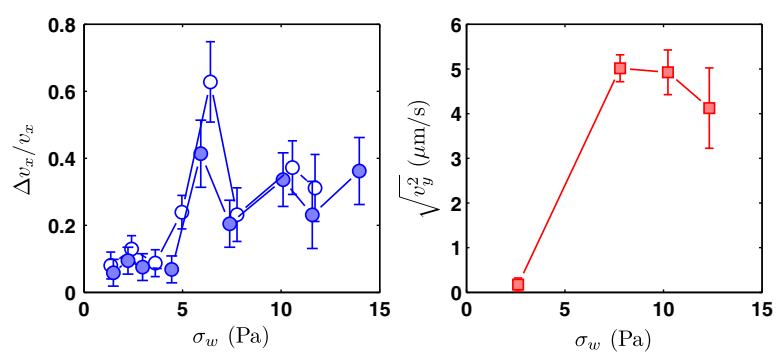

FIG. 3 (color online). Left: normalized standard deviation of the maximum velocity in the flow direction (channel width: colored circles $170 \mu \mathrm{m}$, open circles $152 \mu \mathrm{m}$ ). Right: standard deviation of the transverse component $v_{y}$ of the velocity field.

$\left[\operatorname{std}\left(v_{y}\right)=0\right]$. For $4.6<\sigma_{w}<8 \mathrm{~Pa}$, we observe a periodic variation of the transverse velocity $v_{y}$ as a function of time (Fig. 4, top left). The period equals $1.17 \mathrm{~s}$ for $\sigma_{w}=7.8 \mathrm{~Pa}$. In this regime the tracers' trajectories are strongly correlated and the std of $v_{y}$ is independent of the position along the channel width, at least in the central region. For $\sigma_{w}>8 \mathrm{~Pa}$, the std of the velocity fluctuations saturates at about $4 \mu \mathrm{m} / \mathrm{s}$, as shown in Fig. 3. The tracer trajectories become random with fewer correlations and a rather broad temporal spectrum, qualitatively illustrated in Fig. 4, right. In these experiments, inertial forces are negligible compared to the viscous forces (Reynolds numbers are less than 0.02). The instability is thus of purely elastic origin. The fluid is highly shear thinning; there is thus a strong gradient of normal stress and shear rate close to the wall, which can destabilize the base flow, depending on the fluid properties. As those of the $0.4 \%$ solution are well described by a White-Metzner equation, we could refer to the linear stability analysis proposed by Wilson and Rallison [13], which concludes that the base flow is unstable if the shear thinning exponent is on

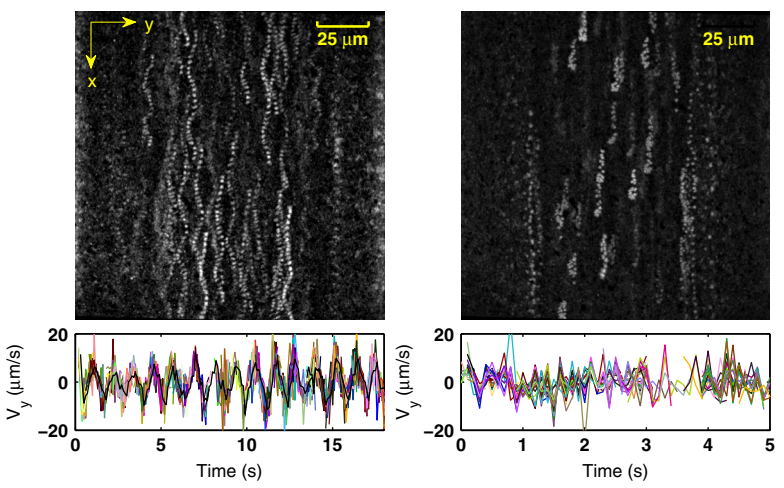

FIG. 4 (color online). Top: superposition of 26 successive images taken at $10 \mathrm{~Hz}$ in the $152 \mu \mathrm{m}$ width channel. The channel was mounted on a translation stage that is moving at a velocity close to the mean liquid velocity, in the $x$ direction. Left: $\sigma_{w}=7.8 \mathrm{~Pa}$, stage velocity $0.83 \mathrm{~mm} / \mathrm{s}$. Right: $\sigma_{w}=12.3 \mathrm{~Pa}$, stage velocity $3.75 \mathrm{~mm} / \mathrm{s}$. Bottom: superposition of all the individual tracer velocity in the $y$ (transverse) direction. Additional pictures and movies are available in the Supplemental Material [16]. the order of or below 0.2 , which is the case of the solution under study. We experimentally found a threshold for the onset of instability for values of Wi between 3.65 and 5.8. We observe that more dilute concentrations exhibit a similar elastic instability occurring for the same value of Wi. The measured values of the critical Wi are $7.3( \pm 3)$ for the $0.1 \%$ solution and $4( \pm 1)$ for the $0.2 \%$ solution (see Supplemental Material [16]). These measurements suggest that the critical Wi does not depend significantly upon the concentration and can be assumed constant and equal to $5( \pm 3)$. This value reasonably compares to the theoretical one $\mathrm{Wi}=2$. The discrepancy may be due to the fact that our fluids are not pure White-Metzner fluids with constant elastic modulii. Theory [13] predicts a temporal period for the velocity oscillations (as observed in Fig. 4) equal to $\pi w / U_{\max } / \omega_{c}$ where $U_{\max }$ is the velocity of the centerline and $\omega_{c}$ is the characteristic dimensionless frequency given by the numerical analysis (near the instability, $\omega_{c}=0.8$ ). For the data displayed in Fig. 4, this leads to a period of $0.72 \mathrm{~s}$ in reasonable agreement with the observed $1.17 \mathrm{~s}$.

We now analyze the impact of the instability on the flow. From the velocity profiles in such a channel, we can deduce the local rheological behavior [27]. As explained above, the time averaged local shear stress field is given by $\sigma_{x y}=2 y \sigma_{w} / w$. The mean local shear rate $\dot{\gamma}=\partial_{y}\left\langle v_{x}\right\rangle$ is directly obtained by differentiating the averaged velocity profiles. Strikingly, for all the experiments above the onset of instability, the local shear stress is significantly lower than the one measured using the rheometer, in stable flow conditions (see Fig. 1). The effective viscosity of the liquid in the unsteady channel flow is thus smaller than the unperturbed one. This point is clearly highlighted in Fig. 2. The expected velocity profiles, calculated using the bulk rheological properties, are given by $v_{x}=v_{s}+$ $\alpha\left[1-(2 y / w)^{1+1 / n}\right]$, where $\alpha=\left(\sigma_{w} / A\right)^{1 / n} w n / 2(n+1)$ and $v_{s}$ is the slip velocity, treated as a fitting parameter. These theoretical profiles coincide with the measured ones below the onset of the instability, but highly underestimate the maximal velocity above. The discrepancy is higher in the vicinity of the threshold. This result indicates the presence of genuine drag reduction associated with the onset of instability and the presence of velocity fluctuations.

In the following we propose a framework capturing this effect. It relies on the observation that the velocity fluctuations exhibit a time scale (typically on the order of $1 \mathrm{~s}$ ) which is slightly shorter than the relaxation time of the liquid (3.6 s at $\dot{\gamma}=1 \mathrm{~s}^{-1}$ ). Thus, the structural properties, such as the shear-rate dependent viscosity, are advected by these fluctuations. Velocity fluctuations exchange fluid parcels initially located close to the center and having high viscosity with parcels located close to the wall with low viscosity. Such a process would have no effect in a Newtonian liquid but tends to homogenize the viscosity in a shear thinning liquid, which is consistent with the rounding of the velocity profiles displayed in Fig. 2. Let us assume that it is the 

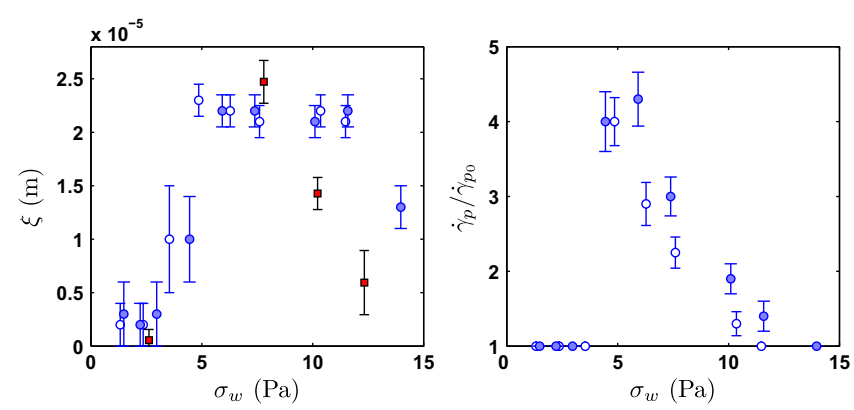

FIG. 5 (color online). Left: characteristic length $\xi$ involved in the nonlocal fluidity model (see text) as a function of the wall shear stress (width of the channel: $152 \mu \mathrm{m}$ open circles, $170 \mu \mathrm{m}$ colored circles). These values are corresponding to the best fit to the mean velocity profiles displayed in Fig. 2. For comparison is displayed (squares) the characteristic length $\tau \sqrt{v_{y}^{2}}$ where $\sqrt{v_{y}^{2}}$ is the amplitude of the transverse velocity fluctuations and $\tau$ the relaxation time of the solution for $\sigma=\sigma_{w} / 2$. Right: Experimental wall shear rate $\dot{\gamma}_{w}$ normalized by the expected value in the absence of instability $\dot{\gamma}_{w_{0}}=\left(\sigma_{w} / A\right)^{1 / n}$.

fluidity $f$, defined by $\dot{\gamma} / \sigma$, which is advected [28]. In stable stationary conditions, as measured by the rheometer, the unperturbed fluidity $f_{0}$ is given by $f_{0}=1 / G \tau$, which is a power-law function of $\dot{\gamma}$. Because of fluctuations, it varies both in space and time. We supplement the classical White-Metzner equation by a generic transport equation for the fluidity which reads $\partial_{t} f+\vec{v} \cdot \vec{\nabla} f=\alpha\left(f-f_{0}\right) . \alpha$ is the inverse of a characteristic time, and should be on the order of $1 / \tau$. We then split the variables into their time averaged values and fluctuations as $f=\langle f\rangle+f^{\prime}$ and $\vec{v}=\langle\vec{v}\rangle+\vec{v}^{\prime}$, where the brackets stand for the mean values and where $\left\langle f^{\prime}\right\rangle=\left\langle\vec{v}^{\prime}\right\rangle=0$. The time average of Eq. (1) reduces to $\left\langle\vec{v}^{\prime} \cdot \vec{\nabla} f^{\prime}\right\rangle=\alpha\left(\langle f\rangle-\left\langle f_{0}\right\rangle\right)$. By analogy with the mixing length theory [29], we propose that $\left\langle v_{i}^{\prime} \partial_{i} f^{\prime}\right\rangle=$ $-\nu_{i i} \partial_{i}^{2}\langle f\rangle$, where $\nu_{i i}$ are positive scalar coefficients that depend on the flow. The symmetry of the mean flow implies that $\langle f\rangle$ depends only upon $y$. This leads to

$$
\xi^{2} \frac{d^{2}\langle f\rangle}{d y^{2}}=\left\langle f_{0}\right\rangle-\langle f\rangle
$$

where $\xi=\sqrt{\nu_{y y} / \alpha}$ is the characteristic length over which viscosity mixing occurs.

In addition to the straightforward condition $\partial_{y}\langle f\rangle=0$ in the middle of the channel, one requires a second boundary condition to solve Eq. (2). The empirical shear rates at the wall $\dot{\gamma}_{w}$ are used to solve this equation and to fit, after integration, the velocity profiles with $\xi$ being the only fitting parameter [30]. The fitted profiles (Fig. 2) are in good agreement with the experimental data (fluidity profiles are displayed in the Supplemental Material [16]). From almost zero below the onset of the instability, $\xi$ reaches a value of about $20 \mu \mathrm{m}$ above the onset (Fig. 5). This value can be compared to the transverse distance traveled by a polymer molecule before relaxation, which is of the order of $\tau\left\langle v_{y}^{2}\right\rangle^{1 / 2}$. As shown in Fig. 5, this quantity obtained from independent measurements captures well the order of magnitude of $\xi$, which reinforces the consistency of our approach.

In this Letter, we have studied the flow of highly shear thinning polymer solutions in straight channels. We have shown an elastic instability occurring above a critical Weissenberg number. The origin of the instability lies in the important normal stress gradients close to the wall. This elastic instability homogenizes the viscosity profiles, increases significantly the shear rate at the wall, and consequently induces genuine drag reduction despite the presence of strong velocity fluctuations.

[1] R. G. Larson, Rheol. Acta 31, 213 (1992).

[2] S. J. Muller, R. G. Larson, and E. S. G. Shaqfeh, Rheol. Acta 28, 499 (1989).

[3] E. S. G. Shaqfeh, Annu. Rev. Fluid Mech. 28, 129 (1996).

[4] A. Groisman and V. Steinberg, Nature (London) 405, 53 (2000).

[5] A. Groisman and V. Steinberg, New J. Phys. 6, 29 (2004).

[6] J. Zilz, R. J. Poole, M. A. Alves, D. Bartolo, B. Levache, and A. Lindner, J. Fluid Mech. 712, 203 (2012).

[7] A. N. Morozov and W. van Saarloos, Phys. Rev. Lett. 95, 024501 (2005).

[8] B. Meulenbroek, C. Storm, A. N. Morozov, and W. van Saarloos, J. Non-Newtonian Fluid Mech. 116, 235 (2004).

[9] T. C. Ho and M. M. Denn, J. Non-Newtonian Fluid Mech. 3, 179 (1977).

[10] D. Bonn, F. Ingremeau, Y. Amarouchene, and H. Kellay, Phys. Rev. E 84, 045301 (2011).

[11] L. Pan, A. Morozov, C. Wagner, and P. E. Arratia, Phys. Rev. Lett. 110, 174502 (2013).

[12] G. Peters, A. M. Grillet, A. C. B. Bogaerds, and F. P. T. Baaijens, J. Non-Newtonian Fluid Mech. 103, 221 (2002).

[13] H. J. Wilson and J. M. Rallison, J. Non-Newtonian Fluid Mech. 72, 237 (1997).

[14] H. J. Wilson and J. M. Rallison, J. Non-Newtonian Fluid Mech. 87, 75 (1999).

[15] P. Nghe, S. M. Fielding, P. Tabeling, and A. Ajdari, Phys. Rev. Lett. 104, 248303 (2010).

[16] See Supplemental Material at http://link.aps.org/ supplemental/10.1103/PhysRevLett.114.028302, which includes Ref. [17], for flow curve measurements, flow stability in cone and plate geometry, device preparation, Bagley correction, measurement of critical Weissenberg numbers, velocity spectra and fluidity profiles.

[17] G. H. McKinley, P. Pakdel, and A. Oztekin, J. Non-Newtonian Fluid Mech. 67, 19 (1996).

[18] C. Masselon, J.-B. Salmon, and A. Colin, Phys. Rev. Lett. 100, 038301 (2008).

[19] F. Pan and A. Acrivos, J. Fluid Mech. 28, 643 (1967).

[20] E. B. Bagley, J. Appl. Phys. 28, 624 (1957).

[21] The size of the depleted zone can be estimated as $v_{s} \eta_{w} / \sigma_{w}$ [22-25], where $v_{s}$ is the slip velocity and $\eta_{w}$ the water viscosity. It comprises between 4 and $120 \mathrm{~nm}$ which is of the order of the mesh size of the polymer estimated to be $70 \mathrm{~nm}$. 
[22] U. S. Agarwal, A. Dutta, and R. A. Mashelkar, Chem. Eng. Sci. 49, 1693 (1994).

[23] H. A. Barnes, J. Non-Newtonian Fluid Mech. 56, 221 (1995).

[24] A. Cuenca and H. Bodiguel, Phys. Rev. Lett. 110, 108304 (2013).

[25] M. D. Graham, Annu. Rev. Fluid Mech. 43, 273 (2011).

[26] J. C. Crocker and D. G. Grier, J. Colloid Interface Sci. 179, 298 (1996).
[27] G. Degré, P. Joseph, P. Tabeling, S. Lerouge, M. Cloitre, and A. Ajdari, Appl. Phys. Lett. 89, 024104 (2006).

[28] A similar approach could be proposed with advection of the viscosity, with no significant differences on the final result.

[29] U. Frish, Turbulence: The Legacy of A.N. Kolgomorov (Cambridge University Press, Cambridge, 1995).

[30] Note that we also use for the integration the already determined empiric slip velocity. 